\title{
On rational isotropic congruences of lines
}

\author{
Boris Odehnal
}

\begin{abstract}
The aim of this paper is to show a way to find an explicit parametrization of rational isotropic congruences of lines in Euclidean three-space $\mathbb{R}^{3}$. It will be shown that also the focal surfaces of these congruences admit a rational parametrization. Furthermore, the close relation of isotropic congruences of lines to minimal surfaces will be used to find the related polynomial minimal surfaces.
\end{abstract}

Mathematics Subject Classification (2000): 53A25, 53A10, 14J26

Keywords: differential line geometry, line congruences, isotropic congruences, rational parametrizations, polynomial minimal surfaces

\section{Introduction}

In this paper we consider the set $\mathcal{L}$ of lines of Euclidean $\mathbb{R}^{3}$ as the four-dimensional Grassmann manifold. The two-dimensional submanifolds $\mathcal{C}$ of $\mathcal{L}$ are called congruences of lines and have been a field of intensive study since the first half of the nineteenth century, see for example [6].

Nowadays congruences of lines have become relevant for practical applications. Motion planing, NC machining and tool path planing are examples where congruences of lines appear. Congruences are studied also from the design viewpoint, see for example [9], [10] and [12].

Among the congruences of lines the so-called isotropic congruences of lines have been of interest, see [5], [13] and [14]. Isotropic congruences of lines also appear in the context of energy minimizing congruences of lines, see [10]. For the design of congruences of lines it is useful to have a geometrically favorable representation. Rational parametrizations are favorable in this sense. The design of curves and surfaces by means of rational parametrizations is well studied, see [4] and [11].

In the following an explicit and rational parametrization of isotropic congruences of lines will be given. Unlike in [5] these parametrizations are free of integrals. For that end the concept of the infinitesimal kinematic mapping introduced by H. R. MÜLLER in [7] will be used to characterize and subsequently parametrize isotropic congruences of lines. 
Afterwards, some results on rational isotropic congruences are shown. The most important among these are:

1. The focal surfaces of rational isotropic congruences admit rational parametrizations. This is surprising since these surfaces appear as the solutions of a quadratic equation.

2. The minimal surfaces appearing as the central envelopes of rational isotropic congruences of lines admit even polynomial parametrizations, if the generating function is a polynomial one. (For detailed explanation see Sec. 4.)

\section{Congruences of lines in Euclidean three-space}

\subsection{Differential geometry of congruences of lines in $\mathbb{R}^{3}$}

The lines of a congruence can be locally parametrized by

$$
L\left(u^{1}, u^{2}\right)=m\left(u^{1}, u^{2}\right)+u^{3} l\left(u^{1}, u^{2}\right),
$$

where $m\left(u^{1}, u^{2}\right): D \subset \mathbb{R}^{2} \rightarrow \mathbb{R}^{3}$ is a parametrization of a director surface and $l\left(u^{1}, u^{2}\right): D \subset \mathbb{R}^{2} \rightarrow S^{2}$ is the field of unit direction vectors of $L$. The spherical image $l(D)$ is a part of the unit sphere $S^{2}$. Changing the director surface $m(D)$ by letting $m^{\prime}\left(u^{1}, u^{2}\right)=m\left(u^{1}, u^{2}\right)+\alpha\left(u^{1}, u^{2}\right) l\left(u^{1}, u^{2}\right)$ with an arbitrary function $\alpha\left(u^{1}, u^{2}\right): D \rightarrow \mathbb{R}$ does not change the lines of $\mathcal{C}$.

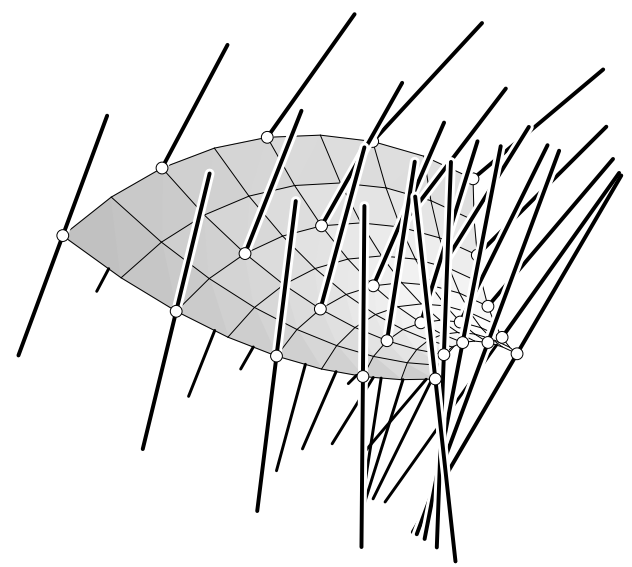

Figure 1: Some lines of a congruence with director surface. 
Now we assign the normalized PLÜCKER coordinates $(l, \bar{l})$ to the lines $L$ in $\mathcal{C}$, see [12]. The first three entries of the PLÜCKER coordinate vector of a line $L$ are simply the coordinates of the unit direction vector $l$. The vector $\bar{l}$ is called moment vector and is defined by $\bar{l}=m \times l$. It is independent on the choice of $m$ on $L$. Its length equals the distance of $L$ to the origin of the coordinate system.

With $\langle\cdot, \cdot\rangle$ we denote the standard scalar product in $\mathbb{R}^{3}$.

Following $[3,6,12]$ we define the two quadratic differential forms

$$
\mathrm{I}=\langle d l, d l\rangle=g_{i j} d u^{i} d u^{j} \quad \text { and } \quad \mathrm{II}=-\langle d m, d l\rangle=\gamma_{i j} d u^{i} d u^{j},
$$

which are called first and second fundamental form of $\mathcal{C}$. For a given direction $\dot{u^{1}}: \dot{u}^{2}$ at $\left(u_{0}^{1}, u_{0}^{2}\right)$ in the congruence the value

$$
u_{s}^{3}=\frac{\gamma_{i j} \dot{u^{i}} \dot{u^{j}}}{g_{i j} \dot{u^{i} \dot{u}^{j}}}
$$

is the $u^{3}$-value of the striction or central point of all ruled surfaces in $\mathcal{C}$ containing $L\left(u_{0}^{1}, u_{0}^{2}\right)$ and having the given direction. Thus the form II is called striction form, see also $[3,6,12]$.

Now we are going to look for the extremal positions of the striction point on a congruence line $L\left(u_{0}^{1}, u_{0}^{2}\right)$. A similar problem is to find the minimum and maximum of the normal curvature of a surface in $\mathbb{R}^{3}$. We have to find the extremal values of II/I. II is in general not symmetric, therefore we symmetrize it by letting

$$
\bar{\gamma}_{i j}:=\frac{1}{2}\left(\gamma_{i j}+\gamma_{j i}\right) \quad \text { and } \quad \overline{\mathrm{II}}:=\bar{\gamma}_{i j} d u^{i} d u^{j} .
$$

This simple minimization problem leads to an eigenvalue problem and the eigenvalues $u_{S_{i}}^{3}$ of $\left(\bar{\gamma}_{i j}\right)\left(g_{i j}\right)^{-1}$ are the extremal values of $u_{S}^{3}$. The corresponding directions of $u_{S_{i}}^{3}$ are called principal directions. If we have $u_{S_{1}}^{3}=u_{S_{2}}^{3}$ at $L$, the line $L$ is called isotropic. Congruences containing only isotropic lines are called isotropic congruences.

We define the central point of a line $L$ in $\mathcal{C}$ as the midpoint of the two extremal positions of the striction points on $L$. The central points form the central surface and can serve as director surface as well, it can degenerate to a curve or a point.

The coordinate functions of the second fundamental form of an isotropic congruence of lines which ist parametrized by its central surface satisfy

$$
\gamma_{11}=\gamma_{22}=0, \quad \gamma_{12}+\gamma_{21}=0,
$$

see $[1,3,12]$. With (5) the computation of principal direction fails and we can say that each direction in an isotropic congruence $\mathcal{C}$ of lines is a principal one. 


\subsection{STUDY's sphere, infinitesimal kinematic mapping}

The normalized PL ̈̈CKER coordinates $(l, \bar{l})$ of a line $L$ satisfy $\langle l, l\rangle=1$ and

$$
\langle l, \bar{l}\rangle=0,
$$

which will be referred to as the PLÜCKER relation. Equation (6) tells us that the moment vectors of lines are tangent vectors of the Euclidean unit sphere described by $l$. Thus the vectors $\bar{l}$ form a vector field on $S^{2}$.

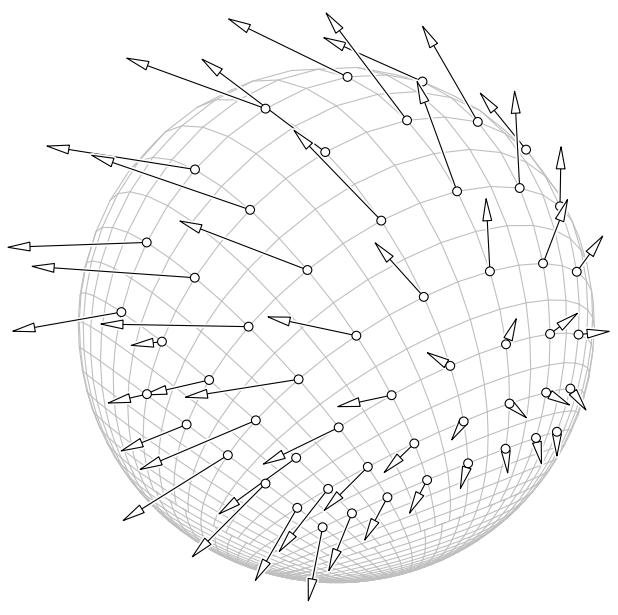

Figure 2: Field of moment vectors on $S^{2}$ of lines of an isotropic congruence.

Following E. STUdy [15], the normalized PLÜCKeR coordinates make it possible to define a mapping $\sigma$ from the set $\overrightarrow{\mathcal{L}}$ of oriented lines in Euclidean three-space to points of the dually extended Euclidean unit sphere $S_{\mathbb{D}}^{2}$, sometimes called STUDY sphere. The orientation of $\mathcal{C}$ 's lines is given by the orientation of $l$. We let

$$
\sigma(L)=l+\epsilon \bar{l}
$$

be the image of the Line $L=(l, \bar{l})$ under the STudy mapping $\sigma: \overrightarrow{\mathcal{L}} \rightarrow S_{\mathbb{D}}^{2}$. The dual unit $\epsilon$ follows the rule $\epsilon^{2}=0$. With (6) we find $\langle\sigma(L), \sigma(L)\rangle=\langle l+\epsilon \bar{l}, l+\epsilon \bar{l}\rangle=$ $\langle l, l\rangle+2 \epsilon\langle l, \bar{l}\rangle=1$ and therefore $\sigma(L)=l+\epsilon \bar{l}$ is a point in $S_{\mathbb{D}}^{2}$.

In [7] the mappings induced by congruences of lines in $S_{\mathbb{D}}^{2}$ are studied. It turns out that these infinitesimal kinematic mappings $L \mapsto \sigma(L)$ determine congruences of lines and vice versa. In [7] H. R. MÜLLER found that an isotropic congruence $\mathcal{C}$ of lines induces an infinitesimal conformal mapping in $S_{\mathbb{D}}^{2}$, if the spherical image of $\mathcal{C}$ 's lines is parametrized isothermally.

Following [7] and assume $l: D \subset \mathbb{R}^{2} \rightarrow \mathbb{R}^{3}$ is an isothermal parametrization of the unit sphere $S^{2}$. The moment vectors $\bar{l}$ of $\mathcal{C}$ 's lines satisfy (6). On the 
other hand, we have $\langle l, l\rangle=1$ and therefore $\left\langle l_{, i}, l\right\rangle=0$, where ${ }_{, i}$ denotes partial differentiation with respect to $u^{i}$. Now the infinitesimal conformal mapping reads

$$
\sigma(L)=l+\epsilon\left(\lambda^{1} l_{, 1}+\lambda^{2} l_{, 2}\right),
$$

where $\lambda^{i}\left(u^{1}, u^{2}\right): D \rightarrow \mathbb{R}$ are real and imaginary part of a holomorphic function $\lambda=\lambda^{1}+i \lambda^{2}$ depending on the complex parameter $u=u^{1}+i u^{2}$. Consequently, $\lambda^{i}$ satisfy the CAUCHY-RIEMANN equations

$$
\lambda_{, 1}^{1}=\lambda_{, 2}^{2}, \quad \lambda_{, 2}^{1}=-\lambda_{, 1}^{2} .
$$

\section{Parametrization of rational isotropic congru- ences}

In order to parametrize rational isotropic congruences of lines, we assume that the unit sphere is isothermally parametrized by

$$
l\left(u^{1}, u^{2}\right)=\frac{1}{N}\left[2 u^{1}, 2 u^{2}, 1-\left(u^{1}\right)^{2}-\left(u^{2}\right)^{2}\right]^{T},
$$

where $N:=1+\left(u^{1}\right)^{2}+\left(u^{2}\right)^{2}$. Since an isotropic congruence $\mathcal{C}$ of lines induces an infinitesimal conformal mapping, the moment vectors of the lines in $\mathcal{C}$ are the dual part of (8) and read

$$
\bar{l}=\lambda^{1} l_{, 1}+\lambda^{2} l_{, 2} .
$$

Now we can state:

\section{Theorem 3.1}

1. Any rational isotropic congruences of lines in Euclidean three-space $\mathbb{R}^{3}$ admits the parametrization

$$
L\left(u^{1}, u^{2} ; u^{3}\right)=\lambda^{1} l_{, 2}-\lambda^{2} l_{, 1}+u^{3} l,
$$

where $l$ is any rational isothermal parametrization of the Euclidean unit sphere $S^{2}$ and $\lambda^{i}$ are real and imaginary part of a rational function $\lambda: D \rightarrow$ $\mathbb{C}$.

2. If a congruence $\mathcal{C}$ can be parametrized by (12), where $l$ is any isothermal parametrization of $S^{2}$ and $\lambda^{i}$ are real and imaginary part of a holomorphic function $\lambda$, then $\mathcal{C}$ is isotropic. 
Proof:

1. Let $\mathcal{C}$ be a rational isotropic congruence of lines. Following [7] $\mathcal{C}$ induces an infinitesimal conformal mapping. Thus the moment vectors $\bar{l}$ of $\mathcal{C}$ 's lines can be described by (11). In order to find a parametrization of $\mathcal{C}$, we compute the surface $P$ of pedal points of $\mathcal{C}$ 's lines. A parametrization of $P$ is given by

$$
P=l \times \bar{l}=l \times\left(\lambda^{1} l_{, 1}+\lambda^{2} l_{, 2}\right)=\lambda^{1} l_{, 2}-\lambda^{2} l_{, 1},
$$

which is the director surface of $\mathcal{C}$ parametrized by (12).

2. By assumption $l$ is an isothermal paramtrization of $S^{2}$, so we have $\langle l, l\rangle=1$, $\left\langle l, l_{, i}\right\rangle=0\left\langle l_{, i}, l_{, j}\right\rangle=\varphi^{2} \delta_{i j}$. Obviously $l_{, 1} \times l_{, 2}=\varphi^{2} l$. With the latter identities we compute the moment vector of the lines described by (12) and arrive at

$$
\bar{l}=\left(\lambda^{1} l_{, 2}-\lambda^{2} l_{, 1}\right) \times l=\lambda^{1} l_{, 1}+\lambda^{2} l_{, 2},
$$

Thus the STUDY image of the lines given by (12) is parametrized by

$$
\sigma(L)=l+\epsilon\left(\lambda^{1} l_{, 1}+\lambda^{2} l_{, 2}\right)
$$

which is an infinitesimal conformal mapping comparable to (8) and (11).

Figure 3 shows some iso-parameter surfaces and some lines of an isotropic congrunece $\mathcal{C}$ of lines. Therein $\lambda=(1+i) u$ is the generating function.

\section{Porperties of rational isotropic congruences}

This section shows some properties of rational isotropic congruences of lines in Euclidean three-space by means of the representation (12).

First we compute the fundamental form (2)

$$
\mathrm{I}=g_{i j} d u^{i} d u^{j}=\varphi^{2} \delta_{i j} d u^{i} d u^{j},
$$

where $\varphi=2 / N$, and the second fundamental form

$$
\mathrm{II}=\gamma_{i j} d u^{i} d u^{j}=-\left\langle m_{, i}, l_{, j}\right\rangle d u^{i} d u^{j} .
$$

The coordinate functions are

$$
\begin{aligned}
& \gamma_{11}=\lambda_{, 1}^{2} \varphi^{2}-\lambda^{1} \varphi_{, 2} \varphi+\lambda^{2} \varphi,{ }_{, 1} \varphi \\
& \gamma_{12}=-\lambda_{, 1}^{1} \varphi^{2}-\lambda^{1} \varphi,{ }_{, 1} \varphi-\lambda^{2} \varphi,{ }_{, 2} \varphi \\
& \gamma_{21}=\lambda_{, 2}^{2} \varphi^{2}+\lambda^{1} \varphi, 1 \varphi+\lambda^{2} \varphi, 2 \varphi \\
& \gamma_{22}=-\lambda_{, 2}^{1} \varphi^{2}-\lambda^{1} \varphi_{, 2} \varphi+\lambda^{2} \varphi,{ }_{, 1} \varphi
\end{aligned}
$$




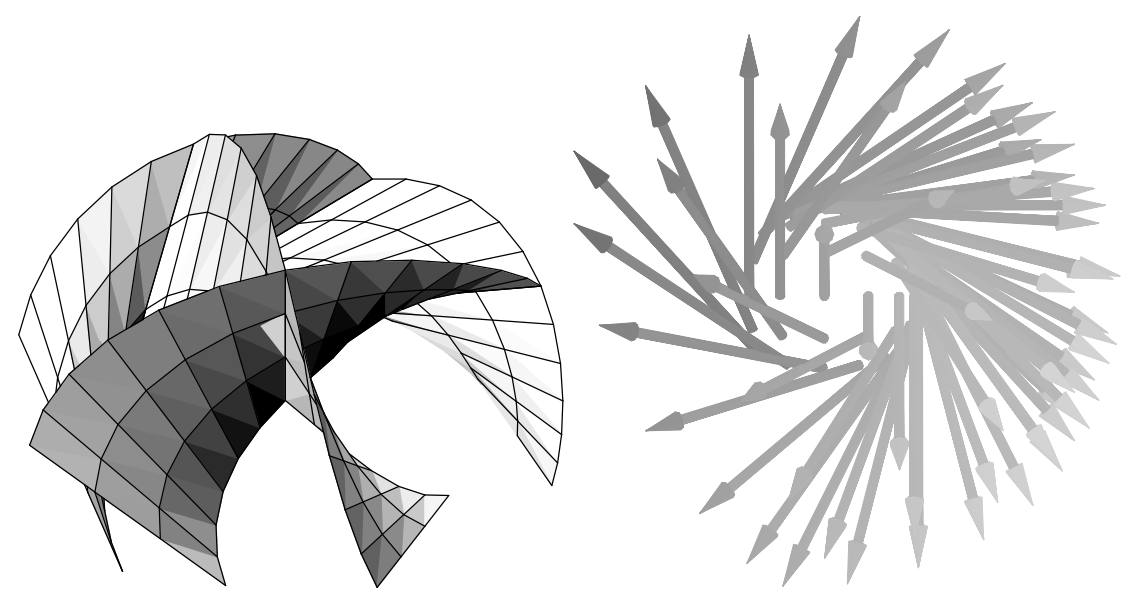

Figure 3: Iso-parameter surfaces (left) and some lines in an isotropic congruence $\mathcal{C}$ (right).

With (3), (14) and (16) we are able to show the following result:

\section{Theorem 4.1}

If $\mathcal{C}$ is a rational isotropic congruence parametrized by (12) and $\lambda: D \subset \mathbb{C} \rightarrow \mathbb{C}$ is a rational function, then the central surface $M$ of $\mathcal{C}$ is a rational surface.

Proof: We compute the central surface $M$ using the formula

$$
u_{M}^{3}=\frac{1}{2} \gamma_{i j} g^{i j}
$$

given in $[3,12]$, where $\left(g^{i j}\right)$ is the inverse matrix to $g=\left(g_{i j}\right)$. Because of (14), (16) and (9), equation (17) simplifies to

$$
u_{M}^{3}=\lambda_{, 1}^{2}-\lambda^{1} \varphi_{, 2} \varphi^{-1}+\lambda^{2} \varphi_{, 1} \varphi^{-1}=: \zeta .
$$

With

$$
M=\lambda^{1} l_{, 2}-\lambda^{2} l_{, 1}+\zeta l
$$

we obtain a rational parametrization of the central surface.

In the following we will use the central surface $M$ as the director surface. Thus we have

$$
L^{\prime}\left(u^{1}, u^{2} ; u^{3}\right)=\lambda^{1} l_{, 2}-\lambda^{2} l_{, 1}+\zeta l+u^{3} l=M+u^{3} l .
$$


Because they are needed later, we compute the coefficients of the second fundamental form of (20). We denote these coordinate functions by $\gamma_{i j}^{\prime}$ and find

$$
\gamma_{11}^{\prime}=\gamma_{22}^{\prime}=0 \quad \& \quad \gamma_{12}^{\prime}=-\gamma_{21}^{\prime}=\gamma_{12}=-\gamma_{21} .
$$

Parametrizations of isotropic congruences of lines can also be obtained by the a theorem given by Ribaucour (1881), see [2]: If $v \in \mathbb{R}^{3}$ and $M$ is the central surface of an isotropic congruence $\mathcal{C}: L=M+u^{3} l$ of lines, then $\mathcal{C}^{\prime}: L^{\prime}=$ $M+v \times l+u^{3} l$ is an isotropic congruence of lines too. The central surfaces of both congruences $\mathcal{C}$ and $\mathcal{C}^{\prime}$ coincide. This leads to further rational congruences of lines:

\section{Theorem 4.2}

If $v \in \mathbb{R}^{3}$ and $M$ is the central surface of a rational isotropic congruence $\mathcal{C}$ (19) of lines, then $\mathcal{C}^{\prime}$ is also a rational isotropic congruence of lines.

\section{Proof:}

1. We do not have to check te isotropy of $\mathcal{C}^{\prime}$, since it is regulated by the above mentioned theorem by RIBAUCOUR.

2. The rationality of $\mathcal{C}^{\prime}$ is obvious: The vector $v$ is a constant vector in $\mathbb{R}^{3}$ and by theorem 4.1 the central surface $M$ of $\mathcal{C}$ and the vector field $l$ are rational.

Using the director surface

$$
M^{\prime}:=M+v \times l
$$

of $\mathcal{C}^{\prime}$ and the identity $\varphi^{2} l=l_{, 1} \times l_{, 2}$ to compute the moment vectors of $\mathcal{C}^{\prime}$ 's lines, we find

$$
\bar{l}^{\prime}=M^{\prime} \times l=M \times l+\langle v, l\rangle l-v=\bar{l}+\langle v, l\rangle l-v .
$$

On the other hand we have the decomposition

$$
\lambda^{1^{\prime}} l_{, 1}+\lambda^{2^{\prime}} l_{, 2}=\bar{l}^{\prime}
$$

and arrive at

$$
\lambda^{1^{\prime}}=\lambda^{1}-\left\langle v, l_{, 1}\right\rangle \varphi^{-2} \quad \text { and } \quad \lambda^{2^{\prime}}=\lambda^{2}-\left\langle v, l_{, 2}\right\rangle \varphi^{-2} .
$$

Thus we can say: 


\section{Theorem 4.3}

Changing the director surface from (12) to the director surface given in (22) the generating function $\lambda^{i}$ change according to (25).

We ask for the singularities of $L$. They are characterized by

$$
F\left(u^{1}, u^{2}, u^{3}\right)=\operatorname{det}\left(L_{, 1}, L_{, 2}, L_{, 3}\right)=0 .
$$

Obviously, $F$ is a quadratic polynomial in $u^{3}$, called focal polynomial, see [3, 6, 12]. Its solutions $u_{F}^{3}$ correspond to the focal points. There are 0,1 or 2 focal points on each line of $\mathcal{C}$. We use the complex extension of $\mathbb{R}^{3}$ and extend $L: D \times \mathbb{R}^{3} \rightarrow \mathbb{R}^{3}$ in the obvious way to $L: D \times \mathbb{C}^{3} \rightarrow \mathbb{C}^{3}$.

It is well known and easy to show that the focal surfaces of isotropic congruences are a pair of conjugate complex developables. The interested reader may find this in the monograph [1].

Indeed, it is a new result that these two developables are rational in the case of a rational isotropic congruence $\mathcal{C}$. So we have the following remarkable result:

\section{Theorem 4.4}

The focal surfaces $F_{1}$ and $F_{2}$ of a rational isotropic congruence $\mathcal{C}$ of lines are a pair of conjuate complex rational developable surfaces.

Proof: We assume $\mathcal{C}$ is parametrized by (20) and use (21) and (26) in order to compute the focal surfaces. So we find

$$
F=\left(u^{3}\right)^{2} \varphi^{2}-\gamma_{12}^{\prime} \gamma_{21}^{\prime} \varphi^{-2}=\left(u^{3}\right)^{2} \varphi^{2}+\gamma_{12}^{2} \varphi^{-2},
$$

which leads to $u_{F 1}^{3}=i \gamma_{12}^{\prime} \varphi^{-2}$ and $u_{F 2}^{3}=-i \gamma_{12}^{\prime} \varphi^{-2}$ with $i^{2}=-1$. The focal surfaces of (20) thus have the parametrization

$$
F_{1}=M+i \frac{\gamma_{12}^{\prime}}{\varphi^{2}} l \quad \text { and } \quad F_{2}=M-i \frac{\gamma_{12}^{\prime}}{\varphi^{2}} l
$$

Comparing (16), (21) and (27), we observe that for any rational generating function $\lambda$ the focal surfaces of $\mathcal{C}$ from (20) are rational.

In the following we use the abbreviation

$$
\mu:=\gamma_{12}^{\prime} \varphi^{-2}
$$

The curves of regression of $F_{i}$ are given by

$$
r_{j}=M \pm i \mu l \pm i\left(\mu_{, 1} \pm i \mu_{, 2}\right) \varphi^{-2}\left(l_{, 1} \mp i l_{, 2}\right),
$$

where $j=1,2$. This can be found in [1]. Now we have the following theorem: 


\section{Theorem 4.5}

The curves of regression of the focal surfaces (27) of rational isotropic congruences $\mathcal{C}$ given by (12) are rational isotropic curves.

Proof: The scalar and vector functions appearing in (29) are rational, if the generating function $\lambda$ is rational, which is immedeatly seen, if we look at (10) and (27).

These curves are isotropic, which means $\left\langle\dot{r}_{j}, \dot{r}_{j}\right\rangle=0$. This is also shown in [1].

The isotropic curves $r_{j}$ of regression of $F_{j}$ are in a close relation to an other surface which is associated to an isotropic congruence $C$ of lines. We call the plane perpendicular to a congruence line $L$ through its central point symmetry plane. The envelope $R$ of the two-parameter family of symmetry planes of $C$ 's lines is called central envelope.

A well known theorem by RIBAUCOUR says that the central envelope $R$ of an isotropic congruence $C$ of lines is a minimal surface, see $[1,3]$. The central envelope admits the parametrization

$$
R=\zeta l+\zeta_{, 1} \varphi^{-2} l_{, 1}+\zeta_{, 2} \varphi^{-2} l_{, 2}=M-\mu_{, 2} \varphi^{-2} l_{, 1}+\mu_{, 1} \varphi^{-2} l_{, 2},
$$

where $\mu$ and $\zeta$ are defined by (28) and (18), respectively. The right hand side of (30) can be found in [1].

In order to show that the right hand side of (30) is a minimal surface, we have to recall S. LIE's generation of minimal surfaces, [8]: Translate an isotropic curve along its conjugate complex counter part. The real points appearing in this motion form a minimal surface.

We use the curves of regression (29), which are isotropic and compute

$$
\frac{1}{2}\left(r_{1}+r_{2}\right)=M-\mu_{, 2} \varphi^{-2} l_{, 1}+\mu_{, 1} \varphi^{-2} l_{, 2} .
$$

The equivalence of the middle term and the right hand side of (30) is shown with help of the CAUCHY-RIEMANN equations (9) and the integrability condition

$$
\varphi\left(\varphi_{, 11}+\varphi_{, 22}\right)-\varphi_{, 1}^{2}-\varphi_{, 2}^{2}+\varphi^{4}=0 .
$$

The integrability condition (31) follows from the fact that $l: D \subset \mathbb{R}^{2} \rightarrow \mathbb{R}^{3}$ from (10) is parametrization of the unit sphere. With the metric (14) induced by $l$ and the theorema egregium we obtain (31).

With the above assumptions on $l$ and $\lambda$ we have:

\section{Theorem 4.6}

The central envelope $R$ of a rational isotropic congruence $C$ (12) of lines is a rational minimal surface. 
Proof: The rationality of $R$ from (30) is obvious, since $\lambda$ is a rational function and $l$ is a rational parametrization of $S^{2}$.

\section{Theorem 4.7}

The central envelope $R$ (30) of a rational isotropic congruence $C$ (12) of lines is a polynomial minimal surface, if the generating function $\lambda$ is a polynomial function $\mathbb{C} \rightarrow \mathbb{C}$.

Proof: Under the assumption that $l$ is parametrized according to (10) we simplify the coordinate functions of (30) and arrive at

$$
R=\left[\begin{array}{c}
\lambda_{, 22}^{1} u^{1} u^{2}+\frac{1}{2} \lambda_{, 11}^{2}\left(1-\left(u^{1}\right)^{2}+\left(u^{2}\right)^{2}\right)+\lambda_{, 1}^{2} u^{1}+\lambda_{, 2}^{2} u^{2}-\lambda^{2} \\
\lambda_{, 22}^{2} u^{1} u^{2}+\frac{1}{2} \lambda_{, 11}^{1}\left(1+\left(u^{1}\right)^{2}-\left(u^{2}\right)^{2}\right)-\lambda_{, 1}^{1} u^{1}-\lambda_{, 2}^{1} u^{2}+\lambda^{1} \\
\lambda_{, 22}^{2} u^{1}-\lambda_{, 11}^{1} u^{2}+\lambda_{, 1}^{2}
\end{array}\right]
$$

Now we assume $\lambda=p(u)$, where $p(u)$ is a polynomial with complex coefficients depending on the complex variable $u=u^{1}+i u^{2}$. The real and imaginary part $\lambda^{1}$ and $\lambda^{2}$ are polynomials too. Inserting them into (32), we find that the coordinate functions of the central envelope are polynomials.

A congruence $C$ of lines in Euclidean space is called normal congruence, if $\gamma_{12}=\gamma_{21}$ independent on the choice of the director surface, see $[3,12]$. These congruences consist of the normals of a surface. We ask if an isotropic congruence can be a normal congruence.

Theorem 4.8 The only rational isotropic congruences of lines that are normal congruences at the same time are the bundles of lines.

Proof: Normal congruences are characterized by $\gamma_{12}=\gamma_{21}$ independently of the choice of the director surface. With (16) and (9) we find the system of differential equations

$$
\begin{aligned}
\left(\lambda_{, 1}^{1}+\lambda_{, 2}^{2}\right) \varphi+\lambda^{1} \varphi_{, 1}+\lambda^{2} \varphi, 2 & =0 \\
\lambda_{, 1}^{1}-\lambda_{, 2}^{2} & =0 \\
\lambda_{, 1}^{2}+\lambda_{, 2}^{1} & =0
\end{aligned}
$$

for $\lambda$. The solutions of (33) belong to isotropic normal congruences. (33) is solved by

$$
\begin{aligned}
& \lambda^{1}=c\left(-1-\left(u^{1}\right)^{2}+\left(u^{2}\right)^{2}\right)+a u^{1} u^{2}+b u^{2} \\
& \lambda^{2}=\frac{a}{2}\left(1-\left(u^{1}\right)^{2}+\left(u^{2}\right)^{2}\right)-2 c u^{1} u^{2}-b u^{1}
\end{aligned}
$$


where $(a, b, c) \in \mathbb{R}^{3}$ are real constants. Inserting (34) into (26), we find

$$
F_{1}\left(u^{1}, u^{2}\right)=F_{2}\left(u^{1}, u^{2}\right)=[-a,-2 c,-b]^{T}=\text { const } .
$$

(35) implies that the point $[-a,-2 c,-b]^{T}$ appears as focal surface, central surface and central envelope of the rational isotropic congruence $C$ generated by $\lambda$ from (34). Therefore the rational isotropic congruences of lines which are normal congruences at the same time are the bundles of lines with vertices (35).

Here we remark that it is easy to show that the bundles of lines are isotropic congruences which are normal congruences at the same time, even if these congruences are not rational.

\section{Examples}

We show examples of low degree rational isotropic congruences of lines and the related low degree minimal surfaces.

We assume that the generating function $\lambda$ of an isotropic congruence $\mathcal{C}$ of lines is a polynomial

$$
\lambda(u)=P u^{4}+Q u^{3}+R u^{2}+S u+T,
$$

where $P, Q, R, S, T \in \mathbb{C}$, not all of them zero and $u=u^{1}+i u^{2}$. With (30) we find that the central envelope is given by

$$
R=\left[\begin{array}{c}
\Im\left(-3 P u^{4}-Q u^{3}+6 P u^{2}+3 Q u+R-T\right) \\
\Re\left(3 P u^{4}+Q u^{3}+6 P u^{2}+3 Q u+R+T\right) \\
\Im\left(-8 P u^{3}-3 Q u^{2}+S\right)
\end{array}\right] .
$$

$R$ is of degree 16 if $P \neq 0$. In the case $P=0$ equation (37) parametrizes EnNePer surfaces, which are of degree 9 as algebraic surfaces.

We use (29) to compute the curves of regression and obtain

$$
r_{1}=\left[\begin{array}{c}
3 i P u^{4}+i Q u^{3}-6 i P u^{2}-3 i Q u-i(R-T) \\
3 P u^{4}+Q u^{3}+6 P u^{2}+3 Q u+R+T \\
8 i P u^{3}+3 i Q u^{2}-i S
\end{array}\right] \text { and } r_{2}=\bar{r}_{1} .
$$

A remarkable example appears if $P=Q=0$.

\section{Theorem 5.1}

The lines of a rational isotropic congruence $\mathcal{C}$ which is generated by a quadratic polynomial can be obtained as the intersection of planes tangent to a pair of isotropic cones. The vertices of these cones are a pair of conjugate complex points. 

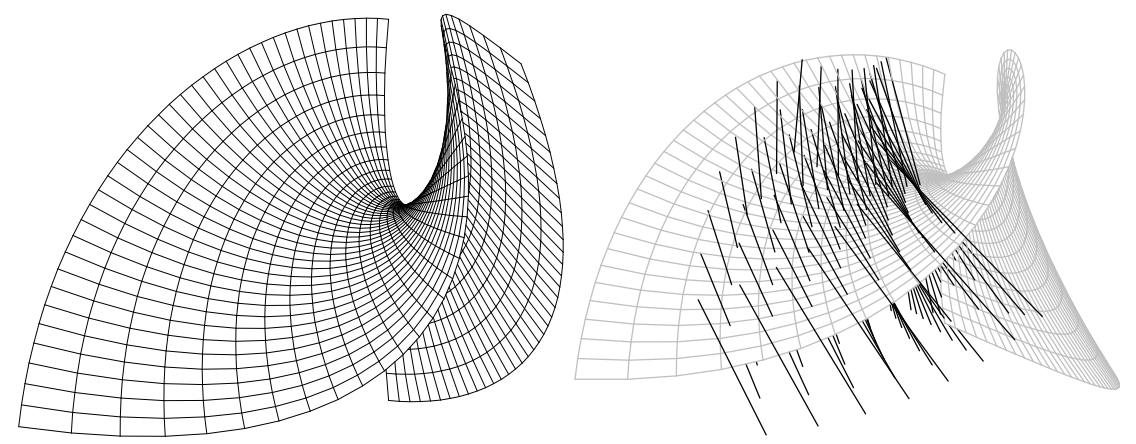

Figure 4: Central envelope (left) and central envelope with some lines (right) of $\mathcal{C}$ defined by $\lambda=u^{4}$.

Proof: In this case the curves given by (38) degenerate to points

$$
r_{1}=[i(T-R), R+T,-i S]^{T} \quad \text { and } \quad r_{2}=\overline{r_{1}} .
$$

The focal surfaces of $\mathcal{C}$ are developables with isotropic generators with points for their curves of regression. Thus the focal surfaces are quadratic cones with vertices $r_{1}$ and $r_{2}$ given by (39), and having the equations

$$
\begin{aligned}
& F_{1}:\left(x^{1}-i(T-R)\right)^{2}+\left(x^{2}-(R+T)\right)^{2}+\left(x^{3}+i S\right)^{2}=0 \\
& F_{2}:\left(x^{1}+i(\bar{T}-\bar{R})\right)^{2}+\left(x^{2}-(\bar{R}+\bar{T})\right)^{2}+\left(x^{3}-i \bar{S}\right)^{2}=0 .
\end{aligned}
$$

In this case the central envelope $R$ also degenerates to a point, being the midpoint of $r_{1}$ and $r_{2}: R=[\Im(R-T), \Re(R+T), \Im S]^{T}$.

\section{Acknowledgements}

I would like to thank J. Wallner for his helpful comments on an earlier version of this paper. I also would like to thank the reviewers for their remarks and suggestions.

\section{References}

[1] W. Blaschke, Vorlesungen über Differentialgeometrie I, Springer Verlag, Berlin, 1921.

[2] G. Bol, Differentialgeometrie III, Skriptum, Freiburg 1971. 
[3] J. Hoschek, Liniengeometrie, Bibliographisches Institut, Zürich, 1971.

[4] J. Hoschek, D. Lasser, Fundamentals of Computer Aided Geometric Design, A. K. Peters, Wellesley, 1993.

[5] R. Koch, Über die Mittelfäche einer isotropen Geradenkongruenz, J. Geometry 23 (1984), 152-169.

[6] E. E. Kummer, Allgemeine Theorie der gradlinigen Strahlensysteme, J. Reine Angew. Math. 57 (1860), 189-230.

[7] H. R. Müller, Über eine infinitesimale kinematische Abbildung, Mh. Math. 54 (1950), 108-129.

[8] J. C. C. Nitsche, Vorlesungen über Minimalflächen, Springer Verlag, Berlin, 1975 .

[9] B. Odehnal \& H. Pottmann, Computing with discrete models of ruled surfaces and line congruences, Proceedings of the $2^{\text {nd }}$ workshop on computational kinematics, Seoul 2001.

[10] B. Odehnal, Geometric Optimization Methods for Line Congruences, Ph. D. Thesis, Vienna University of Technology, 2002.

[11] L. Piegl, W. Tiller, The NURBS Book, Springer Verlag, New York, 1997.

[12] H. Pottmann, J. Wallner, Computational Line Geometry, Springer Verlag, Berlin, 2001.

[13] G. Stamou, Über die isotropen Strahlensysteme, Manuscr. Math. 18 (1976), $15-23$.

[14] N. K. Stephanidis, Minimalfächen und Strahlensysteme, Arch. Math. 41 (1983), 544-554.

[15] E. Study, Geometrie der Dynamen, B. G. Teubner, Leipzig, 1903.

[16] E. A. Weiss, Einführung in die Liniengeometrie und Kinematik, Teubner, Leipzig, 1937.

Boris Odehnal

Institut für Geometrie

Technische Universität Wien

Wiedner Hauptstraße 8-10

1040 Wien, Österreich

e-mail: boris@geometrie.tuwien.ac.at 